\title{
Imaging the effects of peptide materials on phospholipid membranes by atomic force microscopy
}

Katharine Hammond ${ }^{1,2}$, Georgina Benn ${ }^{+1,2,3}$, Isabel Bennett ${ }^{1}$, Edward S. Parsons ${ }^{1}$, Maxim G. Ryadnov, ${ }^{2}$ Bart W. Hoogenboom ${ }^{1,3,4}$, Alice L. B. Pyne ${ }^{1,5 *}$

${ }^{1}$ London Centre for Nanotechnology, University College London, 17-19 Gordon Street, London WC1H OAH, UK.

2 National Physical Laboratory, Hampton Road, Teddington TW11 0LW, UK

${ }^{3}$ Institute of Structural and Molecular Biology, University College London, London WC1E 6BT, UK

${ }^{4}$ Department of Physics and Astronomy, University College London, Gower Street, London WC1E 6BT, UK

${ }^{5}$ Department of Materials Science and Engineering, University of Sheffield, S1 3JD, UK

Email: a.I.pyne@sheffield.ac.uk

\section{Abstract}

Recent advances in biomolecular design require accurate measurements performed in native or near native environments in real time. Atomic force microscopy (AFM) is a powerful tool to observe the dynamics of biologically relevant processes at aqueous interfaces with high spatial resolution. Here we describe imaging protocols to characterise the effects of peptide materials on phospholipid membranes in solution by AFM. These protocols can be used to determine the mechanism and kinetics of membrane-associated activities at the nanoscale.

\section{Running Head}

Imaging peptide materials on membranes

\section{Key words}

Atomic force microscopy, antimicrobial peptides, peptide materials, supported lipid bilayers, phospholipid membranes.

\section{Introduction}

The plasma membrane represents the physical barrier between a cell and its environment. In its most basic form, it comprises a bilayer motif with a thickness of only a few tens of angstroms [1]. The membrane has evolved to selectively allow the passage of ions, small molecules and larger macromolecules in and out the cell. The plasma membrane is also vulnerable to attack, e.g. by pore forming proteins and membrane-targeting peptide assemblies [2, 3]. Particularly notable are antimicrobial peptides that disrupt bacterial membranes, often leading to lysis and cell death [4]. An in-depth understanding of how these peptides interact with membranes can improve the design of more effective antimicrobials demonstrating higher activity and specificity [5].

Recent progress in atomic force microscopy (AFM) has enabled the study of the plasma membrane interface, and its disruption by antimicrobial peptides and peptide nanoscale assemblies in aqueous solution (e.g. [3,6-11]). A straightforward approach to probe the 
impact of peptide treatments on the plasma membrane is to use supported lipid bilayers (SLBs) assembled on a flat mica surface [12]. The lipid composition of SLBs can be tuned to mimic eukaryotic or prokaryotic cell membranes. Bacterial membranes are naturally rich in anionic lipids, phosphatidylethanolamine (PE) and cardiolipin [13]; the most common bacterial membrane mimics consist of mixtures of various zwitterionic and anionic phospholipids. Physiological SLBs form fluid phases and can be prepared from phospholipids with gel-to-fluid transition temperatures that are significantly lower than room temperature [14]. SLBs can be flat to within a few Ångströms, allowing the visualisation of changes to the bilayer surface with nanometre resolution by AFM in aqueous solution [15].

Antimicrobial peptides can interact with phospholipid bilayers in many ways, inducing poration, localised thinning effects and surface roughening [16]. These interactions induce topographical changes which can be distinguished by AFM, as shown in Figure 1.
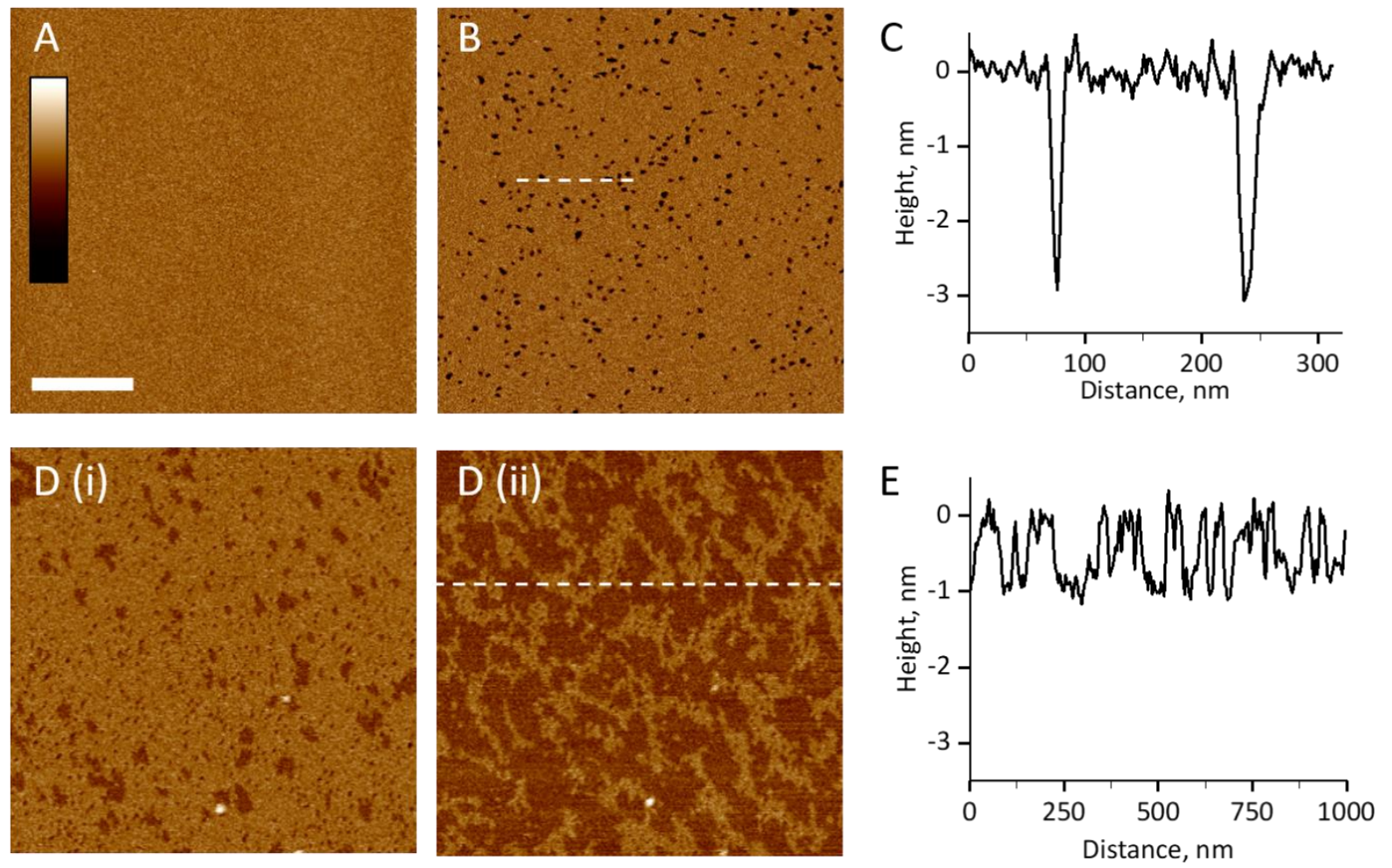

Figure 1: AFM observation of peptide material effects on supported lipid bilayers (SLBs). (A) Topography of an untreated SLB. The surface is featureless and flat to within $<1 \mathrm{~nm}$. (B) Topography of an SLB after addition of a pore-forming antimicrobial peptide. Pores with a diameter of $10-20 \mathrm{~nm}$ (black) can be observed across the surface. (C) Depth profile of the dotted white line in (B), showing two pores with a depth of $3 \mathrm{~nm}$. (D) Dynamic progression of membrane thinning defects (i) $10 \mathrm{~min}$ and (ii) $45 \mathrm{~min}$ after addition of a membrane-thinning peptide. (E) Depth profile of the dotted white line in (D (ii)) showing $1 \mathrm{~nm}$ deep defects in the bilayer. Scale bar: $250 \mathrm{~nm}$. Colour bar: $4 \mathrm{~nm}$.

AFM imaging of membranes treated with peptide should be carried out in fluid to directly observe surface alterations with temporal resolution and minimise potential artefacts due to uncontrolled drying effects. Here we provide a protocol using the rapid force-distance (or offresonance tapping) imaging mode as implemented by Bruker (Santa Barbara, CA), denoted as 
PeakForce Tapping ${ }^{\circledR}$. The protocol also applies to experiments carried out using the more common intermittent contact, or tapping mode, and have also been performed in contact mode (e.g. [11]).

\section{Materials}

Prepare and store all reagents at room temperature, unless indicated otherwise.

\subsection{General Materials}

1. $10 \mathrm{~mm}$ mica substrates (Agar Scientific)

2. $15 \mathrm{~mm}$ magnetic stainless-steel discs (Agar Scientific)

3. Adhesive backed PTFE (Bytac ${ }^{\circledR}$ surface protection laminate)

4. Scalpel or punch

5. Scotch tape

6. Araldite $^{\circledR} 2$-part epoxy resin

7. Stainless steel tweezers

8. Plasma cleaner (Zepto, Deiner Electronics)

2.2. Phospholipids

Phospholipids are purchased from Avanti Polar Lipids (Alabama, USA) as a powder and stored at $-20{ }^{\circ} \mathrm{C}$ prior to use (see Note 1 ).

1. 1-palmitoyl-2-oleoyl-sn-glycero-3-phosphocholine (POPC)

2. 1- hexadecanoyl-2-(9Z - octadecenoyl)-sn-glycero-3-phospho-(1'-rac-glycerol) (POPG)

2.3. Formation of lipid suspension

1. Bath sonicator (Elmasonic PH350EL)

2. 2-5 $\mathrm{mL}$ glass bottles and chloroform safe caps

3. 2,20 , and $200 \mu \mathrm{L}$ Gilson pipettes with $2-20$ and $20-200 \mu \mathrm{L}$ plastic tips

4. $200 \mu \mathrm{L}$ solvent safe pipette tips

5. Extruder (e.g. Avanti Mini-Extruder)

6. $50-200 \mathrm{~nm}$ polycarbonate membranes (Avanti/Whatman)

7. $10 \mathrm{~mm}$ filter supports (Avanti/Whatman)

8. Chloroform

9. Buffer solution: $20 \mathrm{mM}$ HEPES, $120 \mathrm{mM} \mathrm{NaCl}, \mathrm{pH} 7.4$ (See Note 2)

10. Calcium Chloride solution: $100 \mathrm{mM} \mathrm{CaCl}_{2}$ (See Note 2)

\subsection{AFM Cantilevers}

An appropriate cantilever should be chosen from the list below (See Note 3). The cantilever manufacturer, spring constant $(\mathrm{N} / \mathrm{m})$, resonant frequency $(\mathrm{kHZ})$ and nominal tip radius $(\mathrm{nm})$ are provided.

1. FastScan D (Bruker, $0.25 \mathrm{~N} / \mathrm{m}, 110 \mathrm{kHz}, 5 \mathrm{~nm}$ )

2. PEAKFORCE-HIRS-F-B (Bruker, $0.12 \mathrm{~N} / \mathrm{m}, 30 \mathrm{kHz}, 1 \mathrm{~nm}$ )

3. Biolever mini (Olympus, $0.1 \mathrm{~N} / \mathrm{m}, 25 \mathrm{kHz}, 10 \mathrm{~nm}$ )

4. MSNL-E (Bruker, $0.05 \mathrm{~N} / \mathrm{m}, 7 \mathrm{kHz}, 2 \mathrm{~nm}$ )

5. MSNL-F (Bruker, $0.3 \mathrm{~N} / \mathrm{m}, 25 \mathrm{kHz}, 2 \mathrm{~nm}$ )

\section{Methods:}

\subsection{Mica substrates}

1. Cut the adhesive PTFE into circles of the same size as the magnetic steel sample discs (15 $\mathrm{mm})$, using either a punch or scalpel, and adhere to the magnetic discs.

2. Cleave the mica disc using scotch tape, glue to the surface of the PTFE using a small spot of Araldite ${ }^{\circledR}$ 2-part epoxy resin, and leave to cure overnight (Figure 2). 
3. Cleave the mica using scotch tape to obtain an atomically flat, clean surface. Ensure that the final cleave removes an entire layer of mica, to avoid contaminating the surface with residue from the tape.

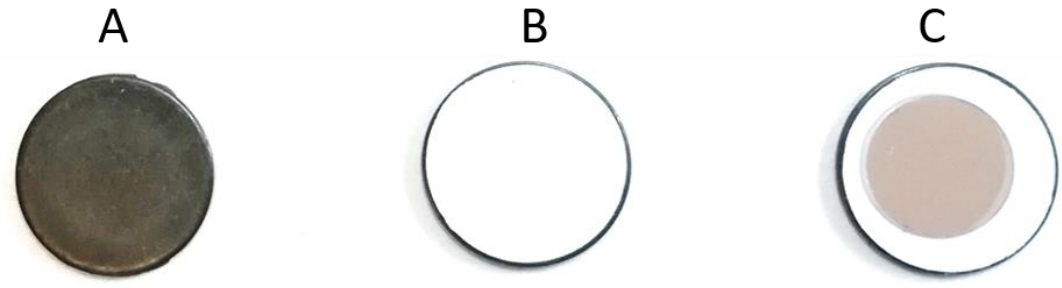

Figure 2: (A) Magnetic steel sample disc (B) PTFE adhered to magnetic steel sample disc and (C) Mica disc glued to PTFE surface using Araldite ${ }^{\circledR}$ 2-part epoxy resin.

\subsection{Formation of Small Unilamellar Vesicles (SUVs)}

1. In a separate glass vial for each phospholipid, weigh out $1 \mathrm{mg}$ of lipid and dissolve in $100 \mu \mathrm{L}$ chloroform to a concentration of $10 \mathrm{mg} / \mathrm{ml}$.

2. In a clean glass vial, combine the volumes of lipid solutions that give the desired molar ratio, and a total mass of $1 \mathrm{mg}$ phospholipid. To form bacterial mimics, zwitterionic and negatively charged lipids are mixed in a 3:1 ratio (see Note 4). For example, to form 3:1 POPC:POPG, combine $75 \mu \mathrm{L}$ POPC solution $(10 \mathrm{mg} / \mathrm{mL})$ and $25 \mu \mathrm{L}$ POPG solution $(10 \mathrm{mg} / \mathrm{mL})$.

3. Dry the lipid-in-chloroform solution under a stream of nitrogen gas to give $1 \mathrm{mg}$ of lipid as a thin film.

4. Hydrate the lipid film in $1 \mathrm{~mL}$ buffer solution, for a final concentration of $1 \mathrm{mg} / \mathrm{mL}$, and vortex to obtain a cloudy vesicle suspension. This is indicative of particulates with a diameter greater than the wavelength of visible light (i.e. $>300-700 \mathrm{~nm}$ ).

5. Sonicate the vesicle suspension in a bath sonicator at $37 \mathrm{kHz}$, at a temperature above the gel to fluid transition temperature of the lipids (see Note 5) until the solution becomes clear. This should take between 5 minutes and 1 hour, depending on the lipid species, the concentration and the position in the bath sonicator (whether the suspension is placed in a sonic 'hotspot').

6. Filter the vesicle suspension using an Avanti mini-extruder through $50 \mathrm{~nm}$ polycarbonate membranes a minimum of 20 times to ensure vesicles of uniform size. This should also be done above the gel to fluid transition temperature of the lipids. The final extruded suspension should be taken from the opposite side to the initial insertion to ensure all vesicles in solution have been passed through the membrane.

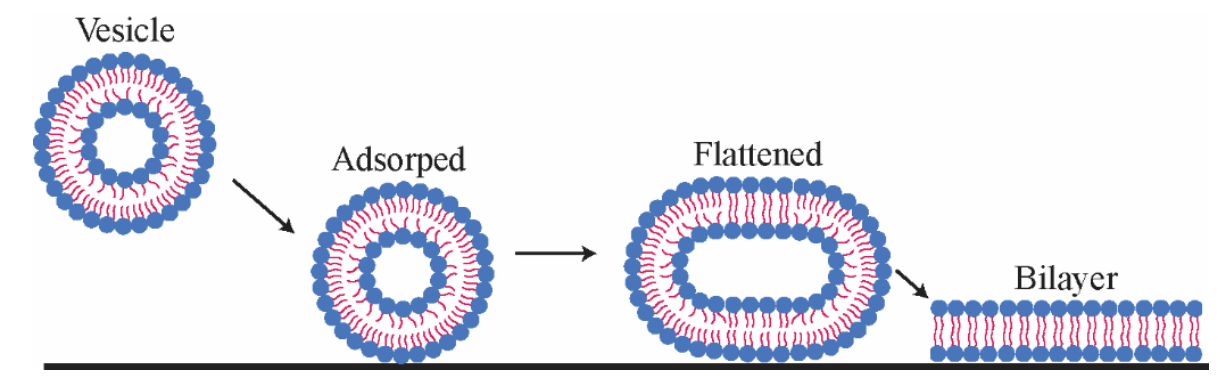

Figure 3. Using the vesicle fusion method, small unilamellar vesicles are adsorbed onto a flat substrate using divalent cations, they then flatten and rupture to form a continuous bilayer. 


\subsection{Formation of a supported lipid bilayer}

Prepare SLBs via the vesicle fusion method (Figure 3) (see Note 6).

1. Add $80 \mu \mathrm{L}$ of buffer solution to a freshly cleaved mica substrate surface, followed by $10 \mu \mathrm{L}$ of the SUV suspension $(1 \mathrm{mg} / \mathrm{ml})$. Finally, add $10 \mu \mathrm{L}$ calcium chloride solution to give a final calcium concentration of $10 \mathrm{mM}$. Calcium aids the adsorption and rupture of the SUVs to the mica surface.

2. Incubate the SUV solution on the mica substrate at room temperature (see note 7) for 30 minutes to allow adsorption and rupture of the vesicles onto the mica surface. Incubation should be performed in a closed or humid chamber, to ensure the surface is kept hydrated throughout (see Note 8).

3. Wash the mica surface thoroughly by adding and removing $50 \mu \mathrm{L}$ of buffer solution 10 times. Take care to ensure the surface is kept hydrated at all times. This will remove excess vesicles from solution, yielding a uniform bilayer free of adsorbed vesicles (as assessed by AFM imaging).

\subsection{AFM imaging of supported lipid bilayers}

1. Prior to use, soak the chosen cantilever in isopropanol:ethanol (1:1) and dry. Then, plasma clean in air for 30 seconds at $10 \%$ power.

2. Mount the chosen cantilever in the AFM and align the laser. Leave the AFM to equilibrate in buffer solution, using a clean freshly cleaved mica disk, during sample preparation.

3. Exchange the blank, equilibration mica disk for the mica disk with supported lipid bilayer on top.

4. Engage the cantilever

5. Imaging parameters will vary, depending on the chosen cantilever. Generally, imaging is carried out at: either 2,4 or $8 \mathrm{kHz}$, with PeakForce amplitudes of $10-20 \mathrm{~nm}$, set-points of $0.05-0.2 \mathrm{~V}(\leq 100 \mathrm{pN})$ and a pixel density of 512 by 512 . More detailed, typical parameters for an MSNL-E are shown in Table 1.

Table 1. Typical parameters used for an MSNL-E cantilever on a Multimode 8 AFM system

\begin{tabular}{|l|l|}
\hline Parameter & Typical value \\
\hline Scan size & $500 \mathrm{~nm}-2 \mu \mathrm{m}$ \\
\hline Aspect ratio & 1 \\
\hline Pixel density & $256 \times 256-512 \times 512$ \\
\hline Line rate & $1-1.5 \mathrm{~Hz}$ \\
\hline Gain & $30-40$ \\
\hline Setpoint & $0.01-0.03 \mathrm{~V}$ \\
\hline Sync Distance & $951120 \mu \mathrm{s}$ \\
\hline PeakForce frequency & $2-4 \mathrm{~Hz}$ \\
\hline Lift height & $7-12 \mathrm{~nm}$ \\
\hline Z range & $1-1.5 \mu \mathrm{m}$ \\
\hline Deflection limit & $12.24 \mathrm{~V}$ \\
\hline
\end{tabular}




\subsection{AFM Force spectroscopy of SLBs}

The flatness of a bilayer, and its similarity to the mica it is deposited on, means that a force curve must be used to confirm its presence. Once the cantilever is engaged a force curve is performed.

1. To begin with, the tip is far enough away from the sample surface (0-75 $\mathrm{nm} \mathrm{Z} \mathrm{distance)}$ that they do not interact.

2. When the tip first comes into contact with the bilayer surface, there is an increase in force as the tip pushes on the bilayer surface, elastically deforming it.

3. When a high enough force is exerted to push the tip through the bilayer, the tip then moves the distance of the bilayer to the mica surface below. This required force, called the breakthrough force, provides information about the stiffness of the bilayer. In addition, the $Z$ distance of this peak can be used to calculate the width of the bilayer.

4. The tip then pushes on the mica substrate, rapidly increasing the force on the cantilever and deflecting the cantilever.

5. The appearance of this characteristic breakthrough force curve allows us to confirm the presence of a bilayer on the mica surface, as this feature is not present in force curves on bare mica. Figure 4 shows the corresponding points on a force curve and as the movement of the cantilever.

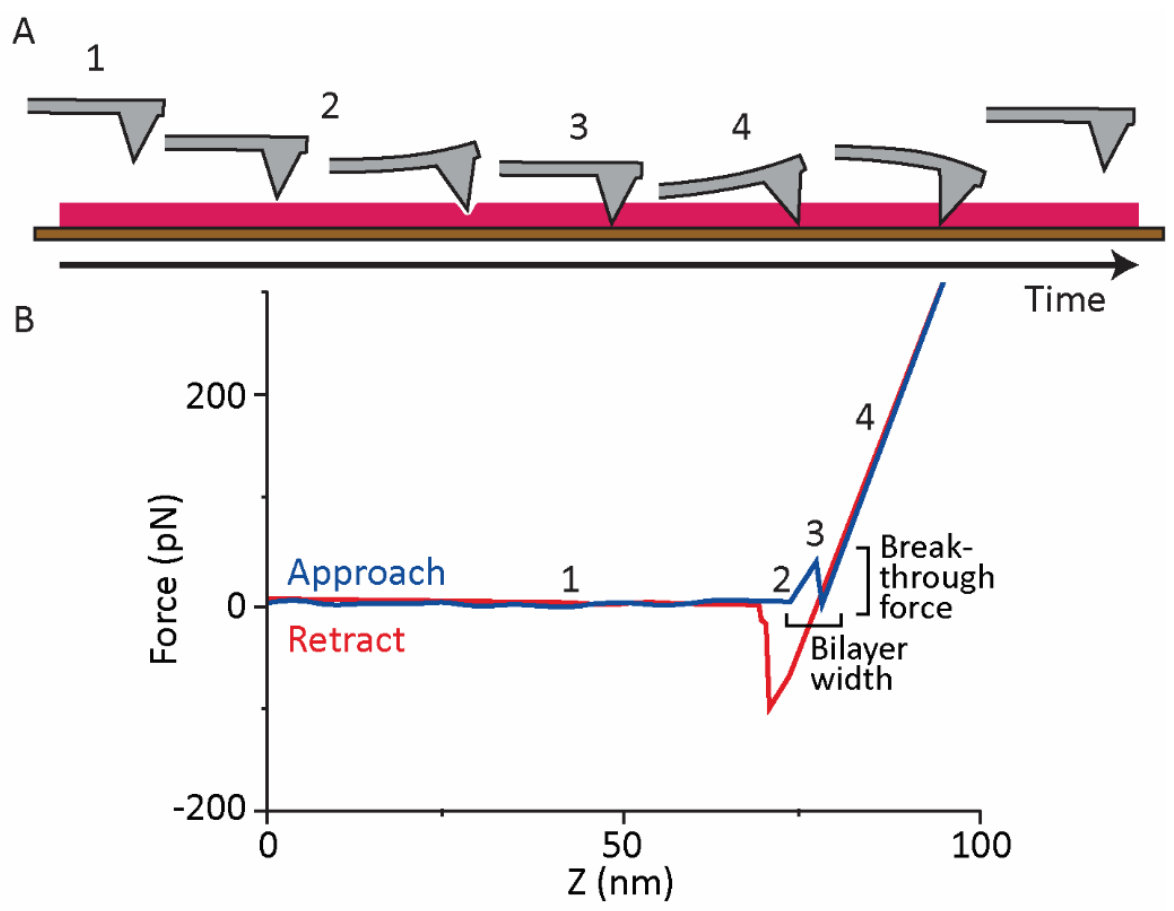

Figure 4: (A) Movement of the cantilever as a force curve is performed on a bilayer (pink) on mica (brown). (B) Force curve showing typical bilayer approach and retract curves for SLB on mica. During approach (blue), the tip breaks through the bilayer to the mica surface, giving rise to a characteristic breakthrough force peak. During retraction (red) there is adhesion of the tip to the bilayer, followed by 'snapping off' and retraction. Numbers correspond to the numbered points in $(A)$.

\subsection{AFM imaging of peptide mechanism of action}

1. After forming a SLB, image to confirm it is free from defects. 
2. Withdraw the AFM tip from the bilayer surface (see Note 9), taking care that the sample remains hydrated.

3. Prepare a $3 \mathrm{mM}$ solution of antimicrobial peptide in buffer.

4. Inject $10 \mu \mathrm{L}$ peptide solution to the lipid bilayer. This will give a final peptide concentration of around $0.3 \mu \mathrm{M}$ (see Note 10).

5. Re-engage the cantilever.

6. Image the peptide treated bilayer until no further changes are observed. This can take between 5 min and 2 hours.

7. Imaging parameters will depend on the cantilever used and the mechanism of membrane disruption, but are typically similar to the imaging parameters used before peptide addition (see Table 1). Optimal scan size will depend on the mechanism observed, but is generally 1-4 $\mu \mathrm{m}$, with 512 pixel density, PeakForce amplitudes of 10$20 \mathrm{~nm}$ and set-points of $0.05-0.2 \mathrm{~V}(\leq 100 \mathrm{pN})$.

6. Once the surface has stopped changing, a larger scan size in the same area should be taken. This is to confirm that the membrane disruption observed is not an artefact due to the forces exerted by the continuously scanning AFM tip. A scan in a different sample location should also be taken (see Note 11).

7. In some cases, peptide can stick to the cantilever and interfere with imaging. Furthermore, peptide-lipid vesicles can form and re-bind to the bilayer surface. To reduce these effects, wait for 15 min after peptide injection (step 3.6.3), before washing the sample surface by adding and removing $50 \mu \mathrm{L}$ of buffer solution 10 times. This allows the peptide to bind to the membrane surface, but removes excess peptide from the imaging solution, enabling cleaner imaging.

4. Notes

1. Other zwitterionic phospholipids such as 1-palmitoyl-2-oleoyl-sn-glycero-3phosphocholine (POPC), 1,2-doileoyl-sn-glycero-3-phosphoethanolamine (DOPE) or 1,2-dioleoyl-sn-glycero-3-phosphocholine (DOPC) may be used instead, or in combination with, DOPC. Other anionic phospholipids could be 1- hexadecanoyl-2-(9Z - octadecenoyl)-sn-glycero-3-phospho-(1'-rac-glycerol) (POPG) or 1,2-dioleoyl-snglycero-3-phospho-(1'-rac-glycerol) (DOPG).

2. It is important to prepare all solutions using ultrapure water (for example, MilliQ ${ }^{\mathrm{TM}}$ which is prepared by purifying deionized water to a resistivity of $18 \mathrm{M} \Omega . \mathrm{cm}$ and TOC $<10 \mathrm{ppb}$ at $25^{\circ} \mathrm{C}$ ) and analytical-grade reagents.

3. The use of an appropriate cantilever is important to obtain adequate resolution, and to minimise damage to the sample. The choice of cantilever depends on the mode of AFM being employed and the type of sample. For imaging of peptide/proteinmembrane interactions in fluid, PEAKFORCE-HIRS-F-B, FastScan D, Biolever Mini, MSNL-E, and MSNL-F cantilevers were selected, due to their low spring constants $(<0.4$ $\mathrm{N} / \mathrm{m}$ ). Additionally, all have a resonant frequency greater than 3 times the frequency they are being driven at in off-resonance tapping.

4. Mixed lipid suspensions are formed by dissolving powdered lipids separately in solvent. Followed by mixing to the correct ratio and drying to a film, this is then rehydrated in buffer. However, for single lipid suspensions or total extracts (e.g. E. coli total lipid extract) steps 3.2.1-3.2.3 may be ignored. 
5. At room temperature, the power generated from sonicating generally heats the bath to approximately $40^{\circ} \mathrm{C}$

6. This technique allows the formation of a continuous bilayer entirely covering the mica. There are no topographic features as the lipids used are miscible and mixed with no phase separation. This gives a flat model system to examine the effect of peptides on the cell membrane.

7. Adsorption must be carried out above the gel-to-fluid transition temperature of the phospholipids used. For all phospholipids listed here, room temperature is sufficient.

8. Care must be taken to ensure that the surface is kept hydrated at all times; dehydration leads to destruction of the supported bilayer as the hydrophilic lipid headgroups do not form an energetically favourable interface with air.

9. The formation of defects in the membrane can be very fast $(<2 \mathrm{~min})$ [10]. To follow the initial formation, in-situ injection should be performed. In this case, after forming an SLB, continue to image the untreated bilayer until the imaging parameters are stable (approximately $10 \mathrm{~min}$ ). Without withdrawing the cantilever or pausing imaging, add $10 \mu \mathrm{L}$ peptide solution to the imaging droplet. This should be done using gel loading pipette tips as they are thinner and therefore easier to inject without disrupting imaging.

10. Depending on the peptide, a higher or lower concentration may be required. It is recommended to try a range of concentrations as peptide-membrane interactions can be concentration dependent.

11. Peptide-membrane interactions can be highly localised and heterogenous across a sample. As such, it is good practice to check different locations of the sample. The same effect should be reproduced across the sample and between repeats before it is considered to be the mechanism of the peptide.

\section{Acknowledgements}

We acknowledge funding by the Engineering and Physical Sciences Research Council (EPSRC: EP/N509577/1; EP/M507970/1; EP/M506448/1; EP/M028100/1), the Biotechnology and Biological Sciences Research Council (BBSRC, BB/N015487/), and the Medical Research Council (MRC, MR/R000328/1; MR/ R024871/1).

\section{References}

1. Garcia-Manyes S, Redondo-Morata L, Oncins G, Sanz F (2010) Nanomechanics of Lipid Bilayers: Heads or Tails? J Am Chem Soc 132:12874-12886 . https://doi.org/10.1021/ja1002185

2. Parsons ES, Stanley GJ, Pyne AL, Hodel AW, Nievergelt AP, Menny A, Yon AR, Rowley A, Richter RP, Fantner GE, Bubeck D, Hoogenboom BW (2019) Single-molecule kinetics of pore assembly by the membrane attack complex. Nat Commun 10:2066 . https://doi.org/10.1038/s41467-019-10058-7

3. De Santis E, Alkassem H, Lamarre B, Faruqui N, Bella A, Noble JE, Micale N, Ray S, Burns JR, Yon AR, Hoogenboom BW, Ryadnov MG (2017) Antimicrobial peptide capsids of de novo design. Nat Commun 8:2263 . https://doi.org/10.1038/s41467017-02475-3

4. Guha S, Ghimire J, Wu E, Wimley WC (2019) Mechanistic Landscape of Membrane- 
Permeabilizing Peptides. Chem Rev 119:6040-6085 .

https://doi.org/10.1021/acs.chemrev.8b00520

5. Li J, Koh J-J, Liu S, Lakshminarayanan R, Verma CS, Beuerman RW (2017) Membrane Active Antimicrobial Peptides: Translating Mechanistic Insights to Design. Front Neurosci 11:73 . https://doi.org/10.3389/fnins.2017.00073

6. Henderson JM, lyengar NS, Lam KLH, Maldonado E, Suwatthee T, Roy I, Waring AJ, Lee KYC (2019) Beyond electrostatics: Antimicrobial peptide selectivity and the influence of cholesterol-mediated fluidity and lipid chain length on protegrin-1 activity. Biochim Biophys Acta - Biomembr 1861:182977 . https://doi.org/10.1016/j.bbamem.2019.04.011

7. Pfeil MP, Pyne AL, Losasso V, Ravi J, Lamarre B, Faruqui N, Alkassem H, Hammond K, Judge PJ, Winn M, Martyna GJ, Crain J, Watts A, Hoogenboom BW, Ryadnov MG (2018) Tuneable poration: host defense peptides as sequence probes for antimicrobial mechanisms. Sci Rep 8:14926 . https://doi.org/10.1038/s41598-01833289-y

8. Pittman AE, Marsh BP, King GM (2018) Conformations and Dynamic Transitions of a Melittin Derivative That Forms Macromolecule-Sized Pores in Lipid Bilayers. Langmuir 34:8393-8399 . https://doi.org/10.1021/acs.langmuir.8b00804

9. Kim SY, Pittman AE, Zapata-Mercado E, King GM, Wimley WC, Hristova K (2019) Mechanism of Action of Peptides That Cause the $\mathrm{pH}$-Triggered Macromolecular Poration of Lipid Bilayers. J Am Chem Soc 141:6706-6718 .

https://doi.org/10.1021/jacs.9b01970

10. Heath GR, Harrison PL, Strong PN, Evans SD, Miller K (2018) Visualization of diffusion limited antimicrobial peptide attack on supported lipid membranes. Soft Matter 14:6146-6154 . https://doi.org/10.1039/c8sm00707a

11. Rakowska PD, Jiang H, Ray S, Pyne A, Lamarre B, Carr M, Judge PJ, Ravi J, Gerling UIM, Koksch B, Martyna GJ, Hoogenboom BW, Watts A, Crain J, Grovenor CRM, Ryadnov MG (2013) Nanoscale imaging reveals laterally expanding antimicrobial pores in lipid bilayers. Proc Natl Acad Sci U S A 110:8918-8923 .

https://doi.org/10.1073/pnas.1222824110

12. Redondo-Morata L, Giannotti MI, Sanz F (2012) Stability of Lipid Bilayers as Model Membranes: Atomic Force Microscopy and Spectroscopy Approach. In: Atomic Force Microscopy in Liquid. Wiley-VCH Verlag GmbH \& Co. KGaA, Weinheim, Germany, pp 259-284

13. Silhavy TJ, Kahne D, Walker S (2010) The bacterial cell envelope. Cold Spring Harb Perspect Biol

14. Nagle JF, Tristram-Nagle S (2000) Structure of lipid bilayers. Biochim Biophys Acta 1469:159-95 . https://doi.org/10.1016/s0304-4157(00)00016-2

15. Morandat S, Azouzi S, Beauvais E, Mastouri A, El Kirat K (2013) Atomic force microscopy of model lipid membranes. Anal Bioanal Chem 405:1445-1461 . https://doi.org/10.1007/s00216-012-6383-y

16. Nguyen LT, Haney EF, Vogel HJ (2011) The expanding scope of antimicrobial peptide structures and their modes of action. Trends Biotechnol 29:464-472 .

https://doi.org/10.1016/j.tibtech.2011.05.001 